\title{
Conjugation and Hyperconjugation Effects: Comparative Studies Through Localized Molecular Orbitals Energy Decomposition Analysis (LMO-EDA) and Natural Bond Orbitals (NBO)
}

\author{
V. A. V. Ferreira, V. G. do Nascimento \& M. B. de Amorim
}

\section{Introduction}

Delocalized chemical bonds are those that are not localized between two atoms only, but three or more atoms. ${ }^{1}$ An example of delocalized chemical bonds is the conjugation effect, measured by energy of delocalization. ${ }^{2}$ In these systems $\mathrm{n} \rightarrow \pi^{*}, \pi \rightarrow \pi^{*}$ or $\pi \rightarrow \mathrm{p}$ (where $\mathrm{n}$ is a lone pair and $\mathrm{p}$ is vacant $\mathrm{p}$ orbital) interactions occur. ${ }^{3}$

Another kind of electronic delocalization is represented by hyperconjugation, which was proposed by Mulliken, and consists on interactions of the $\sigma, \pi$ or $n$ orbitals and a $\sigma^{*}$ orbital. $^{2}$ The hyperconjugation effect is most often overlooked or disregarded by authors, although it is almost ubiquitous. ${ }^{4}$ The most known hyperconjugative effect in the literature is the anomeric effect (negative hyperconjugation $n \rightarrow \sigma^{*}$ ) which is commonly observed in carbohydrates, and whose definition can be extended to structures with a C-X-C-Y pattern (where $\mathrm{X}=\mathrm{N}$, O or $\mathrm{S}$ and $\mathrm{Y}=\mathrm{Br}, \mathrm{Cl}, \mathrm{F}, \mathrm{N}, \mathrm{O}$ or $\mathrm{S}$ ), and the generalized prefix is added to the effect name. ${ }^{5,6}$

These concepts are important to explain properties, stabilization and reactivities of chemical structures, ${ }^{7,89}$ and primarily has its application in organic chemistry and natural products research.

In order to better understand these effects and to apply them in the analysis of chemical and biological properties of natural products, we started the study of methods of analysis of delocalized electron densities.
One of these approaches, the LMO-EDA, ${ }^{10}$ is an variational energy decomposition analysis method based on Morokuma and Kitaura, ${ }^{11,12}$ and Hayes and Stone procedures. ${ }^{13}$ This method uses the localized molecular orbitals of Edmiston and Ruedenberg, which are based on maximum intraorbital repulsion, ${ }^{14}$ for analysis of both covalent bonds and intermolecular interactions. The method decomposes the bond/interaction in contributing terms,

$$
\Delta \mathrm{E}^{\text {Total }}=\Delta \mathrm{E}^{\mathrm{Elect}}+\Delta \mathrm{E}^{\mathrm{Ex}}+\Delta \mathrm{E}^{\mathrm{Rep}}+\mathrm{E}^{\mathrm{Pol}}+\Delta \mathrm{E}^{\text {Disp. }}
$$

whose sum results in the total bond/interaction energy. The first term is related to electrostatic energy, or Coulomb interactions; the second is related to Pauli exchange energy; the third is the Pauli repulsion, caused by orbital superposition; the fourth term is related to polarization energy, and is the sum of charge transfer and bond polarization; and the last term is related to dispersion energy, relative to the difference of the Hartree-Fock (HF) energy and the perturbational methods energy (MPn or CC) obtained for the supermolecule. ${ }^{10}$

Another widely and more reliably method for the study of these effects is NBO, which uses the HF delocalized wave function and the non-orthogonal atomic orbitals (AOs) to obtain the natural atomic orbitals 
(NAOs), the natural hybrid orbitals (NHOs), the natural bond orbitals (NBOs) and the natural localized molecular orbitals (NLMOs), ${ }^{15,16}$

$$
\mathrm{HF}+\mathrm{AOs} \rightarrow \mathrm{NAOs} \rightarrow \mathrm{NBOs} \rightarrow \mathrm{NHOs} \rightarrow \text { NLMOs }
$$

The NBO analysis is based on the natural orbitals of Löwdin (Өi), which have a eigenvalue (ni) correspondent to the maximum occupation of these natural orbitals $(0 \leq$ $\mathrm{ni} \leq 2$, respecting the Pauli exclusive principle), which guarantee the maximum orbital occupation. ${ }^{17}$

An important difference between these methods is the initial orbital set used to the description of the electronic delocalization. ${ }^{4}$ NBO method initially uses orthogonal orbitals, while the LMO-EDA method uses non-orthogonal orbitals that necessarily orthogonalized by the combination of occupied and (higher energy) virtual orbitals, resulting in a exacerbation of the repulsion energy. ${ }^{13,18}$

In order to investigate the usefulness and suitability of the LMO-EDA method in the analysis of the influence of conjugation and hyperconjugation phenomena on chemical structures and to better understand their importance in chemical and biological properties of natural products, herein we started a comparative analysis of the LMO-EDA method with the more reliable NBO method, whose first results are herein reported.

\section{Methods}

The evaluation of the effects previously described made use of appropriate structural models, namely: methoxybenzene (1), where only conjugation effect is found; dimethoxymethane (2), where only hyperconjugation effect exists (Fig. 1).

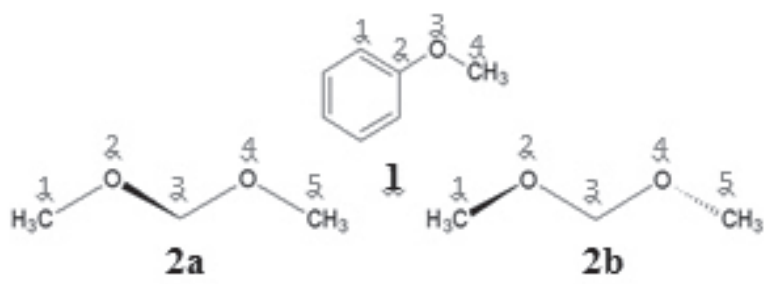

Figure 1: Structures used as appropriate models in this study: 1 methoxybenzene, 2 - dimethoxymetane ( $\mathrm{a}-\mathrm{C} 1$ and $\mathrm{b}-\mathrm{C} 2$ ).
The geometries were optimized and submitted to conformational searches around the $\mathrm{C}-\mathrm{O}$ bonds at the MP2/CC-pVTZ level of theory, both in GAMESS19 and Gaussian0920 packages. The dihedral angles were varied from 0 to $180^{\circ}$, in steps of $10^{\circ}$. The dihedral chosen for varation were (Fig.1): C1-C2-O3-C4, for 1 and $2 \mathrm{a}$ (the last one had its $\mathrm{C} 2-\mathrm{O} 3-\mathrm{O} 4-\mathrm{C} 5$ dihedral kept fixed at $180^{\circ}$ in order to guarantee the presence of only one generalized hyperconjugative effect), and C1-O2$\mathrm{C} 3-\mathrm{O} 4$ and $\mathrm{O} 2-\mathrm{C} 3-\mathrm{O} 4-\mathrm{C} 5$ for $2 \mathrm{~b}$ in order to mantain C2 symmetry and observe the effect of two concurrent hyperconjugative effects. The treatments of wave functions used, LMO-EDA and NBO, were made in the same level theory of the optimization in all conformers obtained in conformational searches.

Energy decomposition analyses were performed using the GAMESS package, while for NBO analysis the ORCA21 package was used.

\section{Results and Discussion}

To analyze how the conjugation and hyperconjugation effects vary and when they occurs we used the models previously shown, methoxybenzene (1) and dimethoxymethane (2) (Fig. 1). As model 2 may have two possibilities of hiperconjugation effect, we locked the $\mathrm{O} 2-\mathrm{C} 3-\mathrm{O} 4-\mathrm{C} 5$ dihedral angle in $180^{\circ}$ of 2 a structure (C1 point group), in order to guarantee the presence of only one generalized hyperconjugative effect.

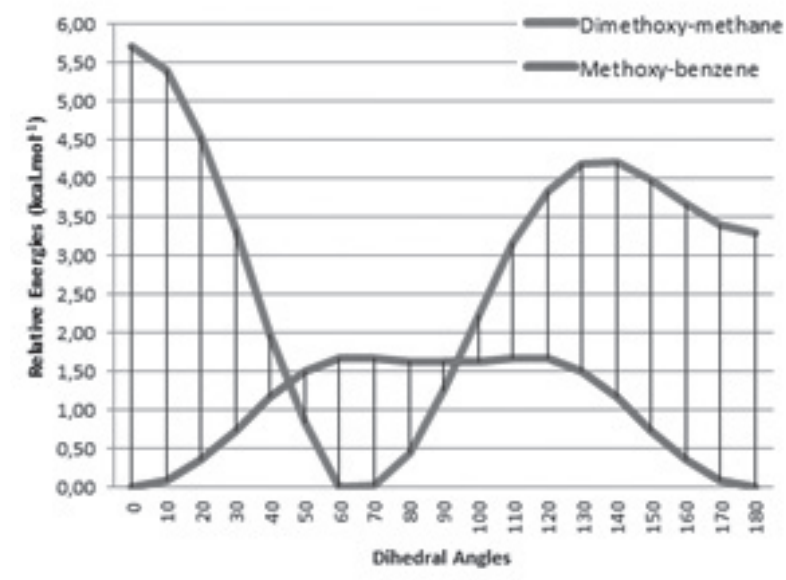

Figure 2: Energy profile of dimethoxymethane and methoxybenzene conformations. 
Figure 2 shows the energy profile of conformational search around the chosen dihedral angle (C1-C2-O3-C4) for 1 and it's equivalent dihedral angle for $2 \mathrm{a}$. For 1 , the most pronounced conjugation interaction occurs in $0^{\circ}$ and $180^{\circ}$ dihedral angles, when the aromatic ring and methoxy group remain in the same plane, allowing a greater orbital overlapping and conjugation effect. For $2 \mathrm{a}$, we notice a greater interaction between $60-70^{\circ}$ dihedral angles $\left(66,98^{\circ}\right.$ with the relaxed geometry), where the orientation of oxygen's lone pair is eclipsed with the $\sigma^{*} \mathrm{C}-\mathrm{O}$, generating an expected greater orbital overlapping and consequent hyperconjugative effect. These hypothesis are evaluated in next steps of this study, where the EDA and NBO analysis are performed.

The LMO-EDA data for 1 and $2 \mathrm{a}$ are shown in figure 3 and 4 , respectively.

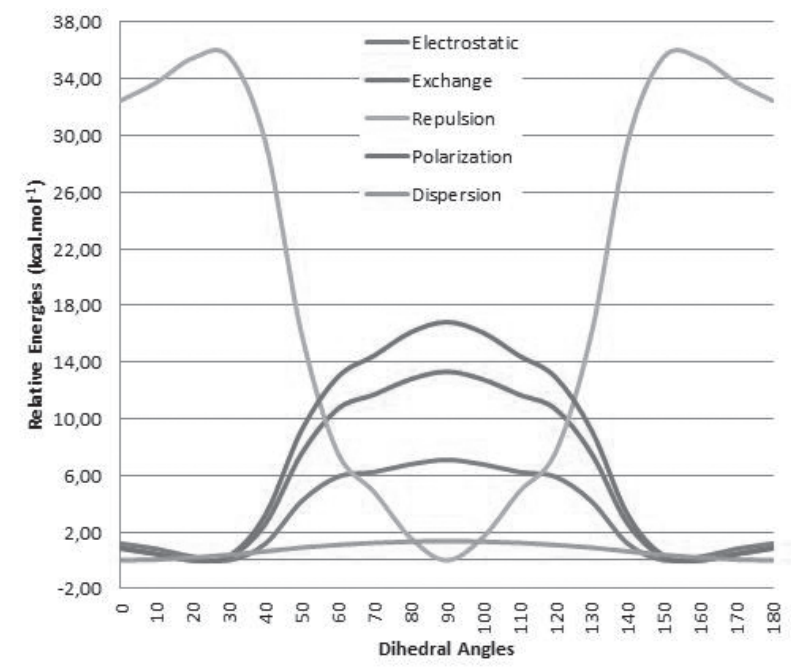

Figure 3: Energy profile of LMO-EDA terms for conformations of 1.

The LMO-EDA profile of 1 (figure 3) shows that in the range of $20-30^{\circ}$ the electrostatic, exchange and polarization terms are stabilizing ones, while the repulsive term is a maximum in energy. At $90^{\circ}$ the repulsive term reaches its global minimum. According to NBO data, conformers shows an interaction between the oxygen's lone pairs (one of the pairs are hybridized sp and the other is purely $\mathrm{p}$ ) with both adjacent aromatic $\pi^{*}$ orbitals which is greater in planar conformation and can probably explains the stabilizing exchange and polarization terms forecasted by LMO-EDA in small dihedral angles.

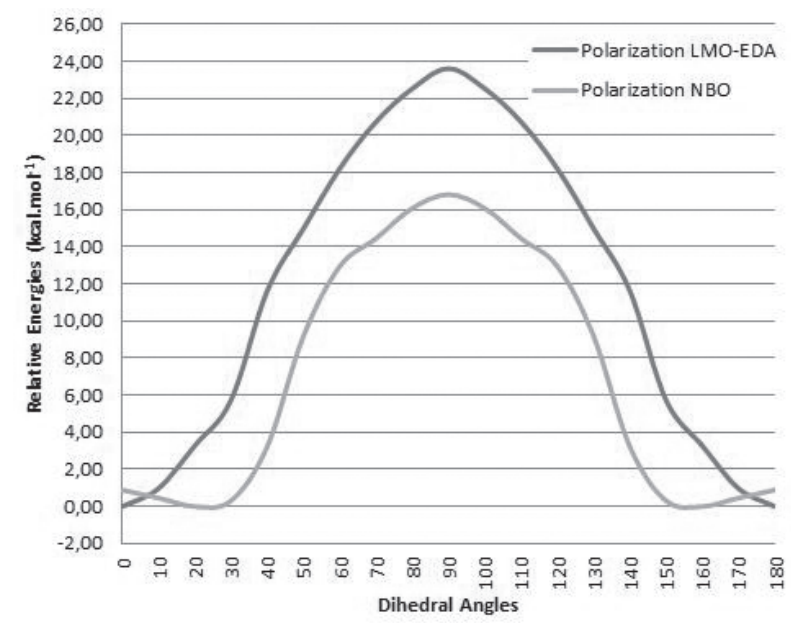

Figure 4. Energy comparative profile of polarization term obtained in LMO-EDA and NBO methods for conformations of 1.

Figure 4 shows the correlation of the polarization terms obtained by both methods for 1 . Within the LMOEDA method the polarization term is obtained by the sum of charge transfer and bond polarization, while for NBO method it was considered by us as the sum of all orbital interaction relative to the analyzed bond. Figure 4 shows a good correlation between them, with exception of the conformers in the range of $20-30^{\circ}$. Nevertheless, an inflexion point is shown in the NBO's profile, indicating a polarization disturb in this range of angles, whose origin we don't know yet.

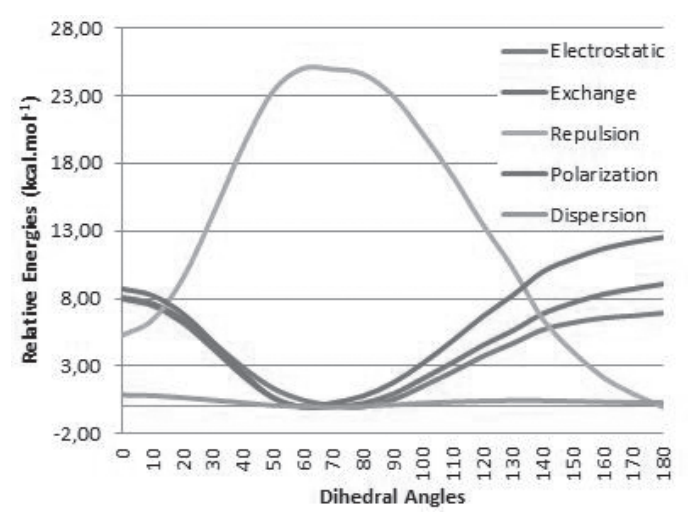

Figure 5. Energy profile of LMO-EDA terms for conformations of 2a. 
For 2a, as shown in figure 5, the LMO-EDA's exchange and polarization terms are stabilizing at $60^{\circ}$, and the electrostatic term has its minimum at $80^{\circ}$. The Pauli repulsion term shows máxima two maxima in energy at this range of dihedral angles.

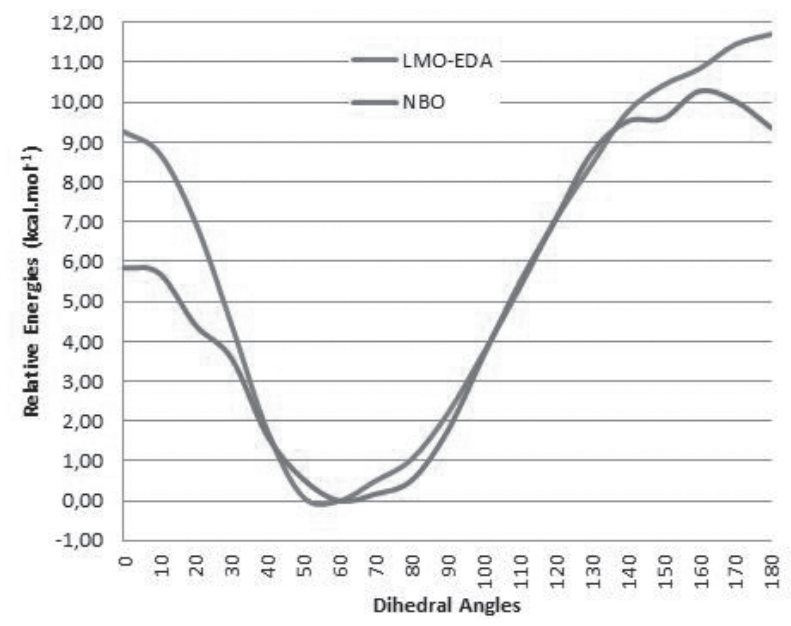

Figure 6. Energy comparative profile of polarization term in LMOEDA and NBO methods for conformations of $2 a$.

Figure 6 shows the correlation of the polarization terms provided by both methods for $2 \mathrm{a}$, where we can see a good correlation between them, except for the values next to planar configuration $\left(0^{\circ}\right.$ and $\left.180^{\circ}\right)$. It must be stressed, however, that the trends are the same for both methods.

The C2 symmetry of dimethoxymetane (2b) may be able to present a second generalized anomeric effect, and therefore important energetic differences (Fig. 7).

Figure 7 shows that the energy profile of $2 b(\mathrm{C} 2$ point group) and of $2 \mathrm{a}$ ( $\mathrm{C} 1$ point group) are very similar, except for the fact that the later has a much more stable gauche (near $60^{\circ}$ ) conformation relative to plane $(\mathrm{C} 2 \mathrm{v})$ ones $\left(0^{\circ}\right.$ and $\left.180^{\circ}\right)$, an indication that both hyperconjugative effects are acting in a cooperative stabilizing way in this $\mathrm{C} 2$ conformer. However, the energy profile obtained by the LMO-EDA method
(Fig. 8) is completely different in comparison with the energy profile of $2 \mathrm{a}$ ( $\mathrm{C} 1$ point group) obtained on the same level. The electrostatic, exchange and polarization terms have an energy minimum near to $40^{\circ}$; while the Pauli repulsion term has a maximum in this same dihedral angle, and a minimum in the opposite situation. Nevertheless, the sum of the contributing terms (including the dispersion one 0 shows that the bond on the conformer with $60^{\circ}$ dihedral angle is stronger than the conformer with $40^{\circ}$.

The comparative profile of polarization terms for $2 \mathrm{~b}$ conformers are shown in figure 9. A great distortion between the conformers on the range of $20-50^{\circ}$ dihedral angle is clearly shown. However, for the others conformers, a good correlations is seen. We believe that this discrepancy occurs due to the presence of a second generalized anomeric effect of same magnitude, but opposite sign, which influences in the bond stabilization, affecting the contributing terms (mainly in the polarization one).

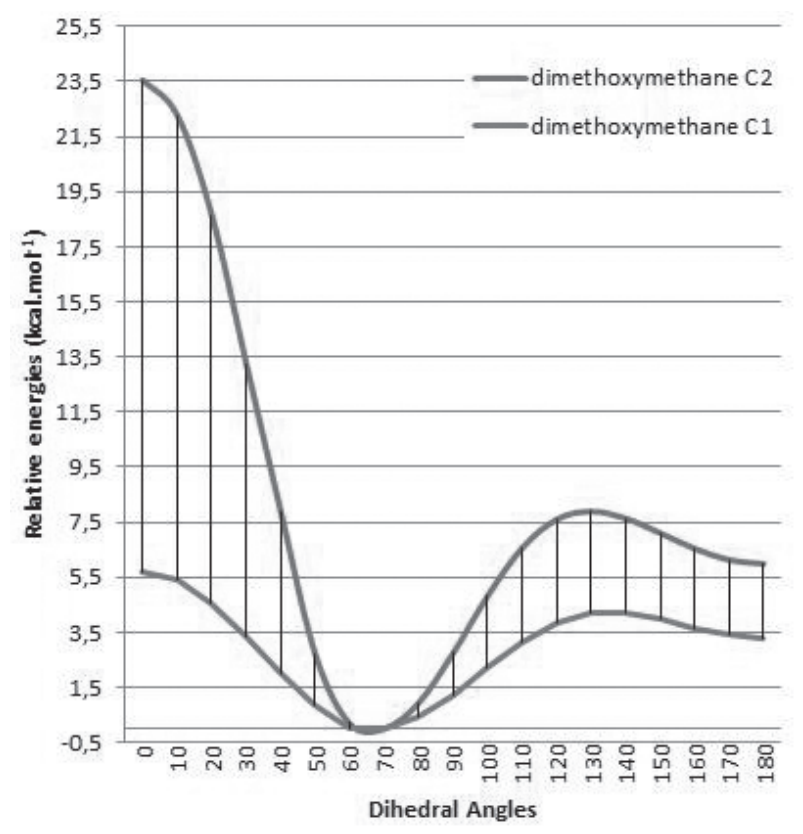

Figure 7. Energy profile of conformations of $2 \mathrm{a}(\mathrm{C} 1$ point group) and $2 \mathrm{~b}\left(\mathrm{C} 2\right.$ point group). Note that in the dihedral angle of $0^{\circ}$ and $180^{\circ}$ the symmetry group of $2 \mathrm{~b}$ becomes $\mathrm{C} 2 \mathrm{v}$. 


\section{Artigo Geral 57}

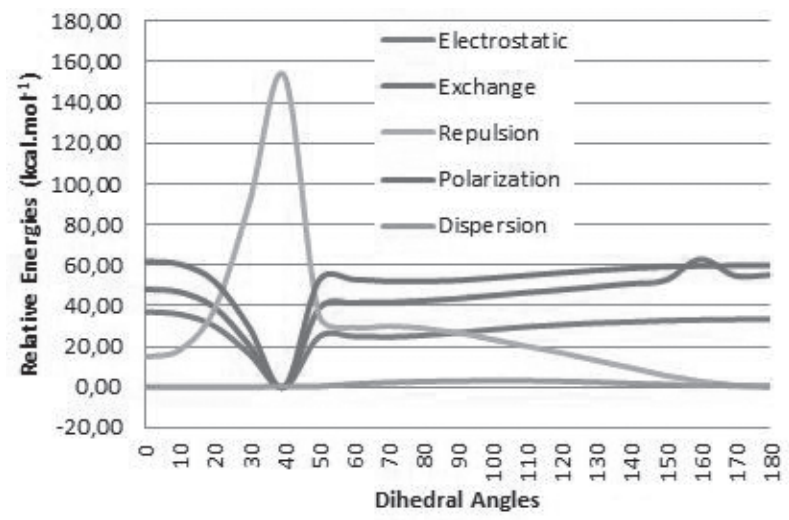

Figure 8. Energy profile of conformations of $2 b$ ( $\mathrm{C} 2$ point group) using the LMO-EDA method.

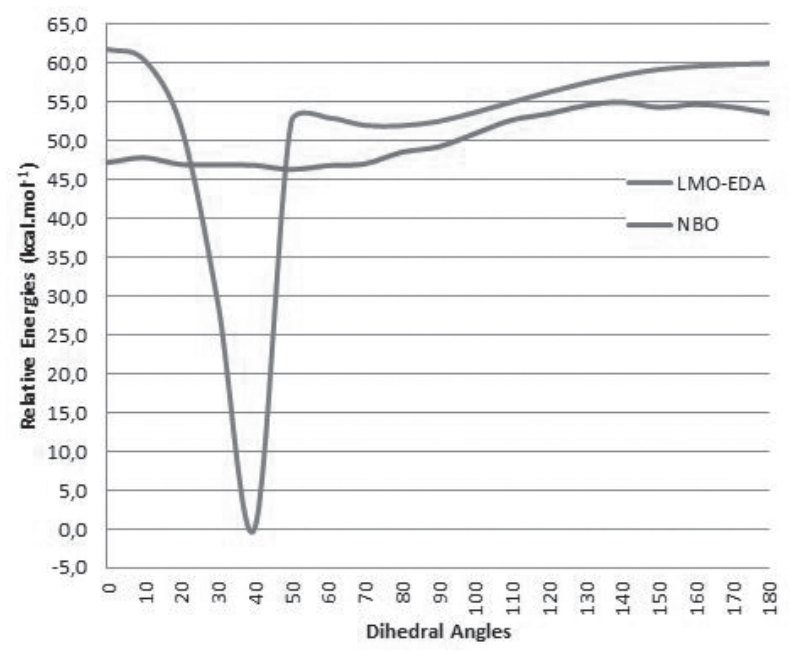

Figure 9. Energy comparative profile of LMO-EDA polarization term and $\mathrm{NBO}$ for $2 \mathrm{~b}$ conformers.

\section{Conclusions}

Despite the Pauli repulsive exacerbation of LMOEDA method, caused by the initial non-orthogonalized orbitals set, the polarization term shows a very good correlation with the NBO polarization, except for the $2 \mathrm{~b}$ structure, which we believe is due to the presence of a second generalized hyperconjugative anomeric effect.
We also believe that LMO-EDA method has a very good description of energies terms involved in bonds, and has proved to be reliable in analyzes of shortrange effects.

In order to do a better comparison of that delocalized effects, we will make the same analysis with the diphenoxymethane structure (same point groups of dimethoxymethane), where the two effects (conjugation and anomeric hyperconjugation) coexist. And then extend that analysis to commonly seen natural products nuclei, 1,2-dimethoxybenzene (competitive conjugative effects) and 1,3-benzodioxole (competitive anomeric hyperconjugative and conjugative effect against each other).

\section{Acknowledgments}

The authors are grateful for the financial support given from the CAPES and CNPQ.

\section{References}

1. M. B. Smith, March's Advanced Organic Chemistry: Reactions, Mechanisms, and Structure, 7th ed., Wiley, (2013).

2. R. S. Mulliken, C. A. Rieke, W. G. Brown, J. Am. Chem. Soc., 63, 41, (1941).

3. IUPAC, The Gold Book, 2nd ed., (2006).

4. I. V. Alabugin, K. M. Gilmore, P. W. Peterson, WIREs Comput. Mol. Sci., 1, 109, (2011).

5. J. T. Edward, Chem. Ind. - London, 1102, (1955).

6. I. Tvaroska \& T. Bleha, Adv. Carbohyd. Chem. Bi., 47, 45, (1989).

7. B. Milián-Medina \& J. Gierschner, WIREs Comput. Mol. Sci., 2, 513, (2012).

8. H Hopf, Wiley-VCH, (2000); R. P. Orenha, R. Vessecchi, S. E. Galembeck, Struct. Chem., 26, 365, (2015).

9. M. Flister \& Q. K. Timerghazin, J. Phys. Chem. A, 118, 9914, (2014).

10. P. Su \& H. Li, J. Chem. Phys., 131, 014102, (2009).

11. K. Kitaura \&, K. Morokuma, Int. J. Quantum Chem., 10, 325, (1976).

12. K. Morokuma, J. Chem. Phys., 55, 1236, (1971).

13. I. C. Hayes \& A. J. Stone, Mol. Phys., 53, 83, (1984).

14. C. Edmiston \& K. Ruedenberg, Rev. Mod. Phys., 35, 457, (1963).

15. J. P. Foster \& F. Weinhold, J. Am. Chem. Soc., 102, 7211, (1980). 
16. A. E. Reed, R. B. Weinstock, F. Weinhold, J. Chem. Phys., 83, 735, (1985).

17. P. Löwdin, Phys. Rev., 97, 1474, (1955).

18. F. Weinhold \& J. Carpenter, J. Mol. Struct. THEOCHEM, 165, 189, (1988).

19. M.W. Schmidt, et al., J. Comput. Chem., 14, 1347, (1993).

20. M. J. Frisch, et al., Gaussian, Inc., Wallingford CT, (2009).

21. F. Neese, WIREs Comput. Mol. Sci., 2, 73, (2012).

\section{A. V. Ferreira*, V. G. do Nascimento \& M. B. de Amorim}

Walter Mors Institute of Research on Natural Products (IPPN), Federal University of Rio de Janeiro (UFRJ).

*E-mail:ferreiravav@correio.nppn.ufrj.br 